\title{
Developmental trajectory of the prefrontal cortex: a systematic review of diffusion tensor imaging studies
}

\author{
Sónia S. Sousa ${ }^{1} \cdot$ Edson Amaro Jr $^{2} \cdot$ Alberto Crego $^{1}$ Óscar F. Gonçalves ${ }^{1,3,4}$. \\ Adriana Sampaio ${ }^{1}$
}

Published online: 15 September 2017

(c) Springer Science+Business Media, LLC 2017

\begin{abstract}
Fluctuations in gray and white matter volumes in addition to the fibers' reorganization and refinement of synaptic connectivity apparently happen in a particular temporo-spatial sequence during the dynamic and prolonged process of cerebral maturation. These developmental events are associated with regional modifications of brain tissues and neural circuits, contributing to networks' specialization and enhanced cognitive processing. According to several studies, improvements in cognitive processes are possibly myelin-dependent and associated to white matter maturation. Of particular interest is the developmental pattern of the prefrontal cortex (PFC), more specifically the PFC white matter, due to its role in high-level executive processes such as attention, working memory and inhibitory control. A systematic review of the literature was conducted using the Web of Science, PubMed and Embase databases to analyze the development of PFC white matter using Diffusion Tensor Imaging (DTI), a widely used non-invasive technique to assess white matter maturation. Both the research and
\end{abstract}

Sónia S. Sousa

soniamachado@psi.uminho.pt

1 Neuropsychophysiology Lab, CIPsi, School of Psychology, University of Minho, Campus Gualtar, 4710-057 Braga, Portugal

2 Department of Radiology, School of Medicine, University of Sao Paulo, São Paulo, Brazil

3 Spaulding Neuromodulation Center, Department of Physical Medicine \& Rehabilitation, Spaulding Rehabilitation Hospital and Massachusetts General Hospital, Harvard Medical School, Charlestown campus: 79/96 13th Street, Charlestown, MA 02129, USA

4 Department of Applied Psychology, Bouvé College of Health Sciences, Northeastern University, 404 International Village, Boston, MA 02115, USA reporting of results were based on Cochrane's recommendations and PRISMA (Preferred Reporting Items for Systematic Reviews and Meta-Analysis) guidelines. Information extracted from 27 published studies revealed an increased myelination, organization and integrity of frontal white matter with age, as revealed by DTI indexes (fractional anisotropy [FA], mean diffusivity [MD], radial diffusivity $[R D]$ and axial diffusivity [AD]). These patterns highlight the extended developmental course of the frontal structural connectivity, which parallels the improvements in higherlevel cognitive functions observed between adolescence and early adulthood.

Keywords Prefrontal cortex - White matter · Myelination · Diffusion tensor imaging $\cdot$ Development

\section{Introduction}

Several studies assessed white matter maturation through non-invasive imaging techniques such as DTI. This technique has become popular considering its sensitivity in evaluating axons' features, using the random motion of water molecules known as thermal Brownian motion (Hagmann et al. 2006; Le Bihan 2003; Thomason and Thompson 2011). This random motion of water molecules is essential to the accuracy of DTI and therefore deserves a detailed explanation of its underlying mechanism. The random displacement observed in biological tissues, seems to be driven by its anatomical architecture, and dependent on the histological properties of tissues such as cell membranes, myelin sheaths and molecules (Hagmann et al. 2006; Mori and Zhang 2006). In this sense, water diffusion within a specific milieu might be classified as isotropic-water molecules travel randomly in all directions, 
or anisotropic-water molecules get displaced into a specific direction (Hagmann et al. 2006; Thomason and Thompson 2011). Once water molecules spread around more freely, the micromotion of the water molecules in gray matter (GM) is considered isotropic. This process is associated to the less fibrillar and intricate nature of GM, when compared to the white matter. In the healthy white matter this process is different and the movement of water molecules is essentially considered anisotropic, or in other words, water molecules travel along a preferred direction, guided by structural barriers such as glial cells, axonal membranes and myelin bundles, present in white matter (Beaulieu 2002; Hagmann et al. 2006; Pierpaoli et al. 1996).

Diffusion-weighted image (DWI) acquisition consists in the application of a gradient sequence within a commonly used range of $700-1300 \mathrm{~s} / \mathrm{mm}^{2}$, values that might change depending on the acquisition protocol. This sequence is applied to the entire brain covering a minimum of six directions in the $\mathrm{X}, \mathrm{Y}$ and $\mathrm{Z}$ axes, in addition to an image with no gradient $\left(b=0 \mathrm{~s} / \mathrm{mm}^{2}\right)$ at least (Alexander et al. 2007; Alger 2012; Chilla et al. 2015; Nishikawa et al. 2013). The b0 image (s) is used to compare the behavior of the water molecules between its initial state, when no gradient was yet applied, and after gradients' application. To estimate the diffusion values from each voxel of the acquired DWI, the eigenvectors and the eigenvalues need to be determined. The main directions of diffusivity or eigenvectors result from estimating the diffusion along three orthogonal directions. The eigenvalues are the diffusion values associated to each main direction. Once determined, the eigenvectors and eigenvalues describe the properties of the diffusion tensor (Hagmann et al. 2006; Le Bihan et al. 2001). The classification of the eigenvalues is as follows, $\lambda_{1} \geq \lambda_{2} \geq \lambda_{3}$, and each one matches one eigenvector. The eigenvector that corresponds to the largest eigenvalue $\left(\lambda_{1}\right)$ represents the principal direction of the diffusion. The diffusion transversal to the main direction is represented by $\lambda_{2}$ and $\lambda_{3}$ The relation between the eigenvalues reveals the diffusion properties within a specific image. If the eigenvalues significantly change from each other, diffusion is assumed to be anisotropic, but if all the eigenvalues are similar, diffusion is presumed to be isotropic. Once the diffusion tensor is obtained, the scalar indexes representing the mean diffusivity and anisotropy within a specific voxel may be calculated.

Several diffusivity-based measures are referred in the DTI research field, however fractional anisotropy (FA), mean diffusivity (MD), axial diffusivity (AD) and radial diffusivity (RD) seem to be the most widely used. The values extracted through these indexes are associated with the motion of water molecules in brain tissues (Alexander et al. 2007; Alger 2012; Hagmann et al. 2006; Le Bihan et al. 2001; Mori and Zhang 2006; Pierpaoli et al. 1996). Specifically, if the relation between the eigenvalues assumes an elongated ellipsoid shape, the diffusion of water is assumed to be anisotropic, as represented by the FA index.

$F A=\sqrt{\frac{3}{2}} \sqrt{\frac{\left(\lambda_{1}-D\right)^{2}+\left(\lambda_{2}-D\right)^{2}+\left(\lambda_{3}-D\right)^{2}}{\lambda_{1}^{2}+\lambda_{2}^{2}+\lambda_{3}^{2}}}$

FA is a scalar measure of the magnitude of diffusion anisotropy in a voxel, derived from a ratio of the principal diffusivities. Basically, this measure is computed through the comparison of each eigenvalue $\left(\lambda_{1}, \lambda_{2}, \lambda_{3}\right)$ with the diffusion coefficient $(D)$. FA represents the degree in which the diffusion of water molecules is anisotropic or unidirectional. The value of FA representing this motion of water molecules into a preferred direction varies between 0 and 1 . Zero represents isotropic diffusion of water molecules with no directional preference, and 1 represents the unidirectional displacement of water molecules or anisotropy.

A second largely used measure is MD, which derives from the mean of the three eigenvalues $\left(\lambda_{1}+\lambda_{2}+\lambda_{3}\right) / 3$, and indicates the overall displacement of water molecules in a voxel, although not providing information about the direction of the movement. Two additional, although less referred in the literature as FA and MD indexes, are $\mathrm{AD}$ and $\mathrm{RD}$. $\mathrm{AD}$ is derived from the largest of the three eigenvalues $\left(\lambda_{1}\right)$ in the $\mathrm{Z}$ or longitudinal axis. This index represents the rate of water diffusion in the direction of greatest diffusion. The greatest diffusion in white matter is usually considered to be parallel to the axonal tracts. RD derives from the average of the second $\lambda_{2}$ and third $\lambda_{3}$ eigenvalues in the $\mathrm{X}$ and $\mathrm{Y}$-axes, representing the diffusion perpendicular to the fiber direction. The diffusion of water molecules perpendicular to the main direction of fibers is usually lower than the diffusion parallel to fibers' orientation (Alexander et al. 2007; Alger 2012; Hagmann et al. 2006; Le Bihan et al. 2001; Mori and Zhang 2006; Pierpaoli and Basser 1996; Thomason and Thompson 2011). A great advantage of DTI is providing rotationally-invariant diffusivity measures, independent of the position of the fibers in space or how the subjects' head was positioned in the scan (Alexander et al. 2007; Alger 2012).

Several methods are used to analyze DTI data. Voxelwise and tract-based spatial statistics (TBSS) are both wholebrain fully automated voxel-based approaches. Voxelwise analysis runs in SPM allowing comparing group means using a Univariate approach in which each voxel is processed separately. TBSS runs in FSL and analyze all the tracts of the brain, comparing each subject to a group mean skeleton derived from a center value of each tract (Smith et al. 2006). Diffusion tensor tractography (DTT) allows the reconstruction of white matter bundles using the diffusion tensor of each voxel. Finally, the region of interest (ROI) method is 
used to assess regions defined à priori by the researcher and the retrieved mean values are compared between subjects (Mukherjee et al. 2008; Froeling et al. 2016).

Interpreting the DTI indexes in the light of brain maturation is not straightforward, however it seems that these water diffusion related measures effectively change during development reflecting underlying tissues' structural changes (Neil et al. 2002). In this sense, some assumptions regarding the hypothetical associations between diffusivity measures and biological mechanisms have been referred in the literature. Commonly anisotropy is associated to the architecture of the white matter myelinated fibers and increased myelination. High values of FA have been associated with enhanced neural connectivity, white matter packing, greater fiber integrity and myelination. Although the presence of myelin is not a key factor for anisotropic diffusion to exist, it may modulate the degree of anisotropy (Beaulieu 2002). In fact, it seems that myelin depletion or damaged myelin significantly changes the measures of anisotropy (Assaf and Pasternak 2008). Specifically, the degree of anisotropic diffusion apparently rises along the neurodevelopmental process of myelination; whereas, reporting from myelin-related disorders such as Multiple Sclerosis (MS) (Preziosa et al. 2017; Roosendaal et al. 2009) or Wallerian degeneration (Xie et al. 2011), decreased anisotropic values are possibly associated to damaged myelin (Neil et al. 2002). As such, changes in FA may detect rapid microstructural changes in the brain such as neuronal plasticity, axonal structure remodeling, fiber density and reorganization (Ding et al. 2013) and activity-dependent myelination mechanisms (Scholz et al. 2009).

While myelination does not appear to be a condition to significantly change FA, it may be important in detecting alterations in MD. It has been observed that the absence of myelin prompts the water displacement to be greater in the perpendicular direction to the axonal bundles, than in the parallel direction (main axis), increasing thus the MD values (Beaulieu 2002). Hence, increased MD values seem to be associated with myelin lesions; whereas low MD values have been associated with white matter integrity since it reflects diminished free diffusion within tissues (Preziosa et al. 2017).

Regarding the $\mathrm{AD}$, this index is thought to reflect the integrity/damage of axons. It has been shown that obstructions to parallel diffusion seem to increase from the breakdown of the longitudinal axonal structure (Beaulieu 2002). In this sense, high $\mathrm{AD}$ values have been associated with intact healthy axons and low AD values have been linked to injured axons (Beaulieu 2002) as, for example, in Wallerian degeneration (Sun et al. 2008).

Finally, the RD appears to be associated with myelination/demyelination processes (Song et al. 2003; Sun et al. 2006). In fact, it seems that the diffusion perpendicular to the axonal tracts is constrained by the interactions of the water molecules with cellular membranes and myelin sheaths. This constriction is possibly causing diffusion to become hindered once water molecules do not move freely in the presence of obstacles (Alexander et al. 2007; Medana and Esiri 2003; Thomason and Thompson 2011). Thus, while transverse diffusivity is diminished in healthy tissues, it seems to increase in the presence of injured axons as in MS and Wallerian degeneration (Roosendaal et al. 2009; Sun et al. 2008; Xie et al. 2011).

According to neuroimaging findings, the human brain presents a specific maturing schedule throughout life. This particular neurodevelopmental timing seems to be related to progressive and regressive neurobiological events that occur at specific ages, reflecting a dynamic and extended developmental process both in terms of structure and cognitive functioning (Giedd 2004; Lenroot and Giedd 2006). Fluctuations of gray and white matter, as measured through morphometric indexes such as volume and cortical thickness, and the reorganization and refinement of synaptic connectivity apparently happen in a particular temporo-spatial sequence since birth until early adulthood, and are associated to regional modifications of brain tissues and neural circuits (Durston et al. 2001; Giedd and Rapoport 2010; Giedd et al. 1999; Giedd 2008; Huttenlocher 1979; Shaw et al. 2008). Accordingly, these findings suggest that particular structural and functional characteristics arise along brain development, eventually showing interdependency with specific cognitive milestones (Bennett et al. 1964; Casey et al. 2000; Paus et al. 1999; Schmithorst et al. 2005).

Specifically, a major increase of global gray matter (GM) is observed during childhood possibly associated to a high rate of synapse production, and is followed by a decline throughout adolescence (Durston et al. 2001; Giedd et al. 1999; Giedd 2004; Gogtay et al. 2004) This GM thinning during adolescence, which is associated to pruning, among other cellular maturational processes, has been associated to the networks' specialization (Giedd 2004; Lenroot and Giedd 2006; Petanjek et al. 2011) and to improved cognitive skills. This process of GM pruning seems to occur in the frontal lobes between adolescence and early adulthood (Durston et al. 2001; Giedd et al. 1999; Giedd 2004; Gogtay et al. 2004; Lebel and Beaulieu 2011; Lenroot and Giedd 2006; Petanjek et al. 2011).

On the other hand, whole brain white matter generally increases throughout life, fact associated with the growing need of additional and effective inter-neuronal connections to sustain the increasing central nervous system (CNS) complexity (Bennett et al. 1964; Dubois et al. 2013; Durston et al. 2001; Giedd et al. 1999; Giedd 2004; Lenroot and Giedd 2006; Snaidero and Simons 2014).

Myelin, the major white matter compound, enfolds axons and basically acts as an electrical insulator that speeds 
impulse conduction between neurons and increases brain efficiency (Fields 2005, 2008; Snaidero and Simons 2014). The process of axons' myelination during brain development seems to be region-specific, eventually following a specific temporal sequence during the CNS maturation. This axonal myelination process seems to underlie the interaction between brain structure and environmental factors, which conversely modulate brain functioning (Dubois et al. 2013; Fields 2005; Snaidero and Simons 2014).

Actually, it seems that the major myelin formation is an on-going process during the first three to four decades of the human lifecycle, following an inferior to superior and posterior-to-anterior (Colby et al. 2011; Paus 2010) and central-to-peripheral direction of maturation (Dubois et al. 2013; Snaidero and Simons 2014). Specifically, myelination is first observed in the dorsal sensorimotor areas (temporo-occipital and parieto-frontal areas) while more time is needed for the temporo-parietal and frontal association areas to be fully myelinated, probably due to its relation with complex cognitive processes (Deoni et al. 2011; Dubois et al. 2013; Gogtay et al. 2004; Lenroot and Giedd 2006; Paus et al. 2001). Specifically, the prefrontal cortex (PFC) maturation, including the dorsolateral PFC and orbitofrontal PFC, which is not finished before the second decade of life (Giedd 2004; Gogtay et al. 2004) progresses from a posterior to anterior direction (Colby et al. 2011; Gogtay et al. 2004) with the most caudal areas maturing earlier than the rostral ones (Tames et al. 2010).

Additionally, the PFC maturational processes, including the major frontal tracts (superior longitudinal fasciculus; inferior fronto-occipital fasciculus; and uncinate fasciculus) are likely to be associated with the PFC functional role in supporting high-level cognitive functions such as attentional processing, working memory and inhibitory control (Kraus et al. 2007; Martino et al. 2013) suggesting that frontal white matter maturation might be the most relevant neural substrate of improved executive processes.

Finally, changes in white matter organization have been broadly observed, in vivo, through diffusion tensor imaging (DTI) metrics, which are believed to be quite sensitive to myelin changes, providing insight into subtle microstructural alterations (e.g. axons' myelination or demyelination; axonal injury or degeneration; axonal packing), whether these alterations happen during the healthy developmental pathway of the brain or due to clinical conditions (e.g. multiple sclerosis (MS), Wallerian degeneration) (Aung et al. 2013; Fox et al. 2011; Le Bihan et al. 2001; Song et al. 2003; Sun et al. 2006; Xie et al. 2011). Once DTI provides information about axonal organization and integrity of neural pathways that ensure communication among several regions of the brain, which could not be retrieved from standard MRI measures (e.g. volumes or cortical thickness), it may provide relevant findings about PFC white matter developmental course.
Therefore, the main purpose of the present review was to systematize current knowledge, on the developmental pathway of the frontal cortex in terms of its microstructural connectivity, as shown by DTI metrics, using the PRISMA guidelines - http://www.prisma-statement.org - (Moher et al. 2009).

\section{Methods}

A search in peer-review publications was performed in March 2017 using the Web of Science, PubMed and Embase databases to identify relevant papers that evaluated the development of the frontal white matter in healthy individuals, using DTI. A total of 53 articles were identified through database searching. Records were retrieved using the key-expressions: "("Diffusion tensor" OR "DTI") AND (typical OR normal OR healthy) AND (brain development AND "white matter" AND frontal)". In detail, the search through Embase database was performed as follows: advanced search in "Journals", in the fields of Neuroscience and Medicine, using the "key-expressions" in all fields, open access articles, short communications and articles in press; search through Web of Science and PubMed was performed using the "key-expressions". No time restriction was applied. Additional articles that were referenced in other studies were also assessed. In order to pursue the database searching and the assessment for eligibility some inclusion and exclusion criteria were formerly defined. Specifically, articles were included if: 1) were published in a peer-review journal; 2) assessed the frontal white matter development in healthy subjects employing cross-sectional or longitudinal designs; 3) used DWI acquisitions and DTI measures; 4) evaluated one or more out of the four DTI indexes: FA, $\mathrm{MD}, \mathrm{AD}$ and RD; 5) were written in English. As exclusion criteria was established: 1) research using clinical samples; 2) studies correlating DTI measures with clinical variables; 3) other imaging method that was not DTI; 4) and aging studies including only adults ( $>40$ years old). After a careful selection of 53 studies, 12 duplicates were removed, 41 were assessed for eligibility and only 27 articles were included in this systematic review (see the diagram in Fig. 1).

Table 1 lists all the articles revised and provides the first author, year of publication, as well as brief information concerning the participants enrolled in the study in addition to details related to the methodology, regions of interest, DTI indexes, main results and statistical analyses.

\section{Results}

Overall, the data gathered in this systematic review document a growing of FA over the distinct periods of 
Fig. 1 Flow diagram for paper selection
Records identified through database searching

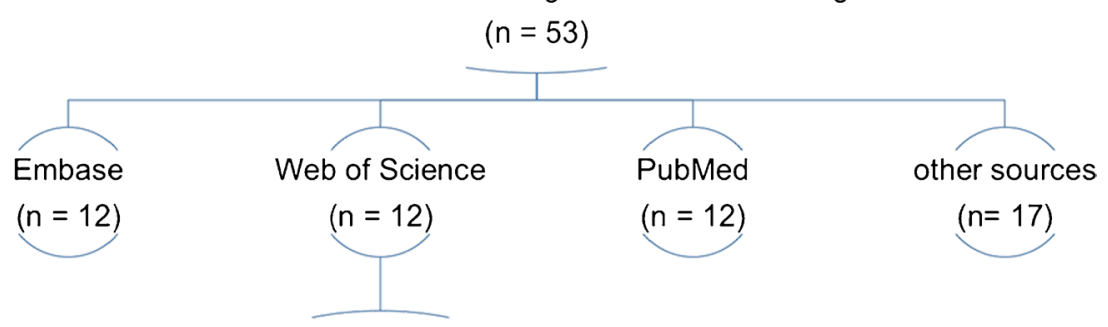

Records after duplicates removed

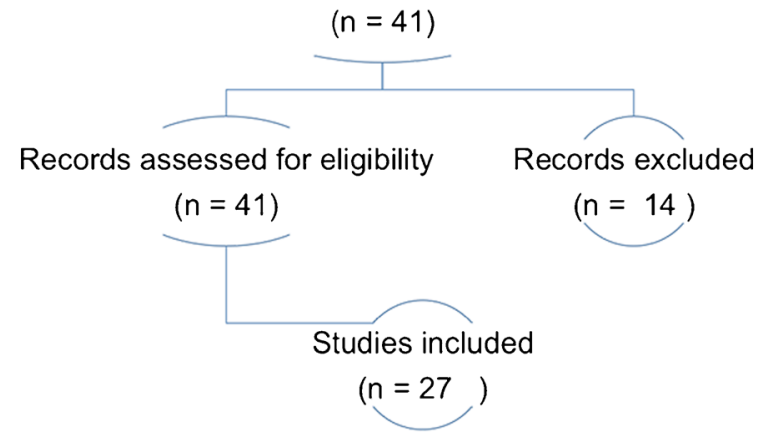

development, concomitant with a reduction of MD and RD and to a lesser extent $\mathrm{AD}$, in the frontal cortex and in its main association tracts. The findings disclosed by the analysis of the DTI indexes in healthy individuals, from neonatal ages until adulthood, suggested a late process of frontal white matter maturing, which still remains in progress throughout the third decade of life.

The majority of the studies that evaluated the FA index in the frontal white matter showed a linear anisotropic increase with age, since the neonatal period until early adulthood (range: birth-30 years), being the lowest FA values observed in children and the highest in young adults (Colby et al. 2011; Schneider et al. 2004; Simmonds et al. 2014). Studies comparing distinct age groups reported that children (range: 8-13) showed the lowest FA values in comparison to older adolescents (range: 16-18) or young adults (range: 18-31, Klingberg et al. 1999; Qiu et al. 2008, 2010), yet no FA changes were observed between younger (range: 6-8) and older children (range: 9-11, Qiu et al. 2008). Additionally, no significant correlations were found between age and FA values in a cohort ranging in age between 5 and 19 years old (Bonekamp et al. 2007).

In terms of the diffusivity measures (MD, RD and $\mathrm{AD}$ ) more variability was observed among studies. In relation to $\mathrm{MD}$, most of the studies that assessed this index showed similar results, i.e. decreasing MD with age (range: 5-43, Bartzokis et al. 2012; Bonekamp et al. 2007; Qiu et al. 2008, 2010) and the greatest trough was observed around 43 years old (Bartzokis et al. 2012); although, a previous study found that infants (range: 24-36 months) displayed similar MD to adults (Schneider et al. 2004).
Regarding the $\mathrm{RD}$ an $\mathrm{AD}$ indexes, the findings revealed an overall decline with age (range: 6-54, Bartzokis et al. 2012; Kumar et al. 2012; Qiu et al. 2008; Simmonds et al. 2014), with the maximum being observed at approximately 35 years old for RD and at age 54 for AD (Bartzokis et al. 2012); whereas, decreased RD but no changes in AD over age (range: 5-28) were observed in the study conducted by Colby et al. (2011).

In the analysis of specific frontal regions-the superior, the middle and the inferior frontal gyri, the dorsolateral and the orbitofrontal cortex - has emerged a pattern of increasing anisotropy and decreasing diffusivity levels along the distinct developmental periods. FA levels were observed to increase with age between the postnatal ages and early adulthood in the superior frontal gyrus (range: 21 days-27 years, Lobel et al. 2009; Moon et al. 2011; Snook et al. 2005; Tamnes et al. 2010), in the middle frontal gyrus (range: 6-35, Barnea-Goraly et al. 2005; Li and Noseworthy 2002; Tamnes et al. 2010), in the inferior frontal gyrus (range: 21 days-30 years, Ashtari et al. 2007; Barnea-Goraly et al. 2005; Lobel et al. 2009; Tamnes et al. 2010), in the dorsolateral, and in the orbitofrontal cortex (range: 6-30, BarneaGoraly et al. 2005; Tamnes et al. 2010). Overall, the studies documented that adults displayed the highest FA levels whether compared with adolescents or children or infants.

Considering the diffusivity indexes MD, RD and AD, a trend for values to decrease with age was observed in the superior frontal gyrus (range: 21 days-30 years, Lobel et al. 2009; Moon et al. 2011; Snook et al. 2005; Tamnes et al. 2010), in the middle frontal gyrus (range: 8-30, Tamnes et al. 2010), in the inferior frontal gyrus 


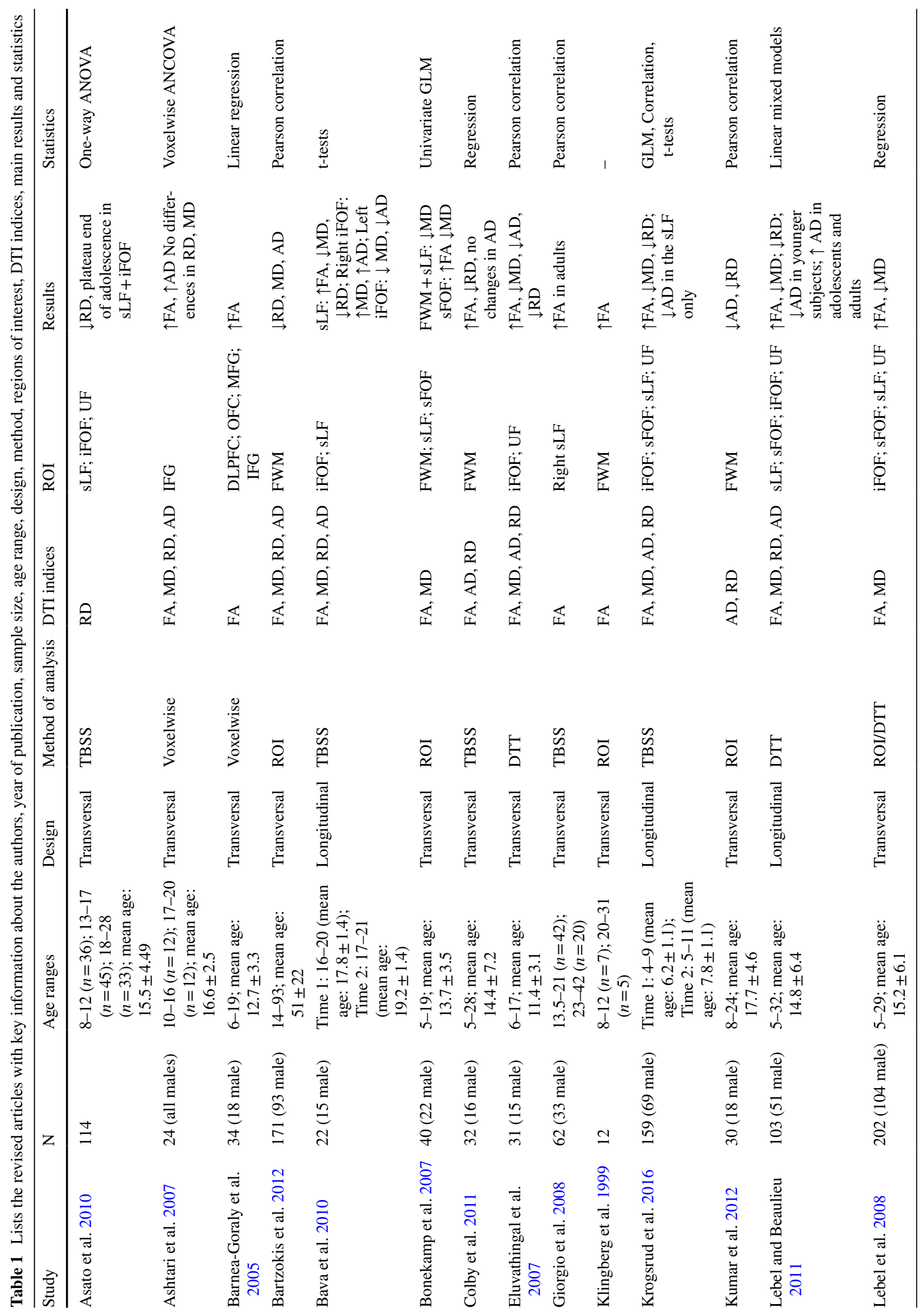




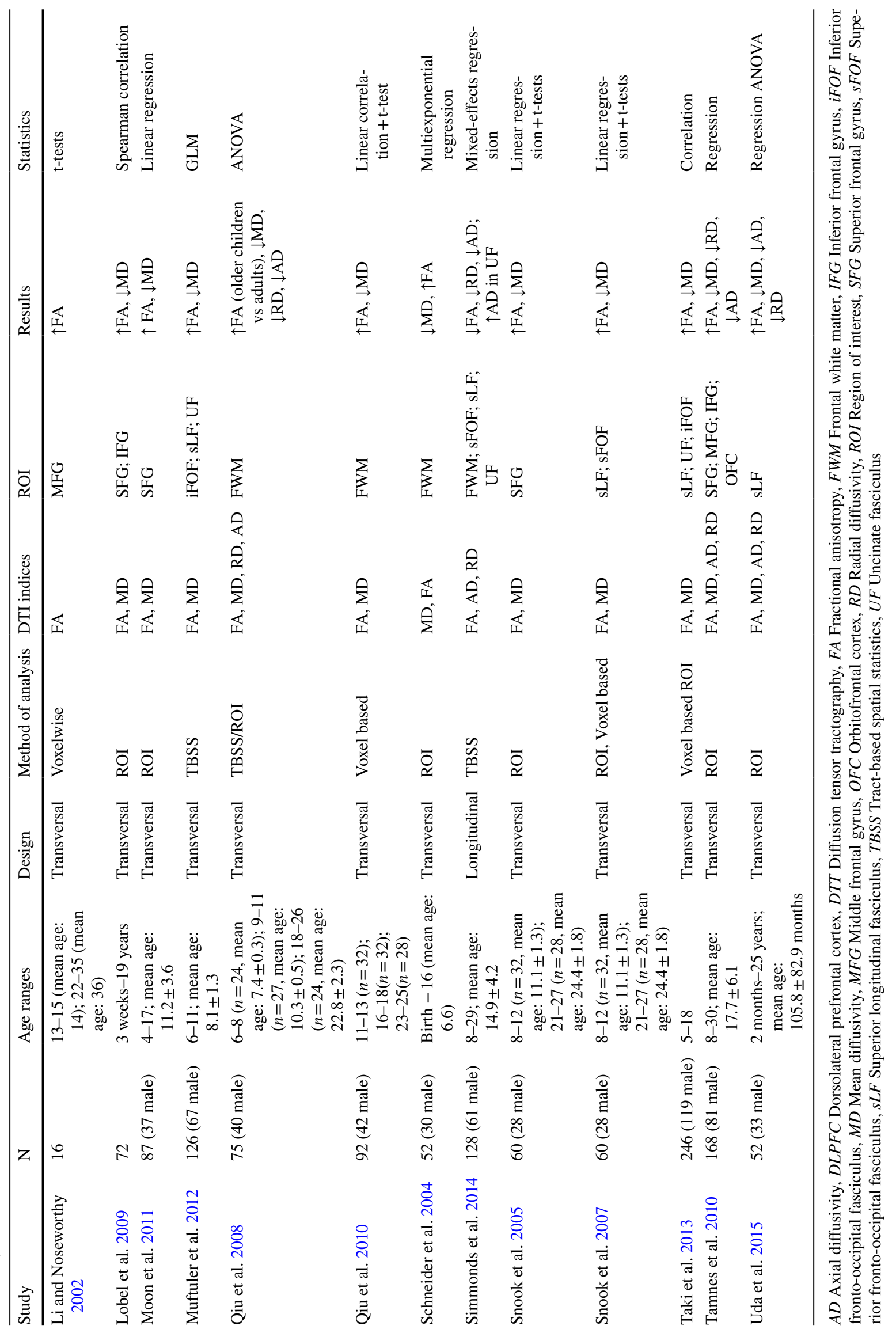


(21 days-30 years, Lobel et al. 2009; Tamnes et al. 2010) and in the orbitofrontal cortex (range: 8-30, Tamnes et al. 2010). Overall, all studies but one (Ashtari et al. 2007) documented that adult individuals displayed the lowest diffusivity levels (MD, RD or $\mathrm{AD}$ ) when compared with adolescents or children or infants. Specifically, Ashtari et al. (2007) found an augmentation of AD without significant changes in RD and MD in the inferior frontal gyrus of older adolescents (range: 17-20) compared to their youngest peers (range: 10-16).

A tendency to an increased FA and decreased MD and $\mathrm{RD}$ as a function of age was also observed in the frontal association tracts, but not so consistent were the results for AD. Specifically, increased FA with age was observed since early ages until adulthood in the inferior fronto-occipital fasciculus (range: 4-30, Eluvathingal et al. 2007; Krogsrud et al. 2016; Lebel and Beaulieu 2011; Lebel et al. 2008; Muftuler et al. 2012; Taki et al. 2013), in the superior frontooccipital fasciculus (range: 4-30, Bonekamp et al. 2007; Krogsrud et al. 2016; Lebel and Beaulieu 2011; Lebel et al. 2008; Snook et al. 2007; Simmonds et al. 2014), in the superior longitudinal fasciculus (range: 2 months-42 years, Bava et al. 2010; Giorgio et al. 2008; Krogsrud et al. 2016; Lebel and Beaulieu 2011; Lebel et al. 2008; Muftuler et al. 2012; Snook et al. 2007; Simmonds et al. 2014; Taki et al. 2013; Uda et al. 2015) and in the uncinate fasciculus (range: 4-30, Eluvathingal et al. 2007; Krogsrud et al. 2016; Lebel and Beaulieu 2011; Lebel et al. 2008; Muftuler et al. 2012; Simmonds et al. 2014; Taki et al. 2013). Conversely, no age-related changes in the FA values were found between childhood and late adolescence (range: 5-19) in the superior longitudinal fasciculus (Bonekamp et al. 2007) and between late-adolescence and early-adulthood (range: 16-21) in the inferior fronto-occipital fasciculus (Bava et al. 2010).

Concerning the diffusivity measures observed in the association tracts, the findings were not so consistent among studies, especially in relation to the rate of change of RD and $\mathrm{AD}$ with age. Specifically, decreased MD values across age in the inferior fronto-occipital fasciculus were found by nearly all the studies included in this review (range: 4-30, Eluvathingal et al. 2007; Krogsrud et al. 2016; Lebel and Beaulieu 2011; Lebel et al. 2008; Muftuler et al. 2012; Taki et al. 2013), excluding the one of Bava et al. (2010), which found inter-hemispheric variations. Likewise, decreasing MD was found in the superior fronto-occipital fasciculus (range: 4-30, Bonekamp et al. 2007; Krogsrud et al. 2016; Lebel and Beaulieu 2011; Lebel et al. 2008; Snook et al. 2007), in the superior longitudinal fasciculus (range: 2 months-30 years, Bava et al. 2010; Bonekamp et al. 2007; Krogsrud et al. 2016; Lebel and Beaulieu 2011; Lebel et al. 2008; Muftuler et al. 2012; Snook et al. 2007; Taki et al. 2013; Uda et al. 2015) and in the uncinate fasciculus (range: 4-30, Eluvathingal et al. 2007; Krogsrud et al. 2016; Lebel and Beaulieu 2011; Lebel et al. 2008; Muftuler et al. 2012; Taki et al. 2013).

A reduction of $\mathrm{RD}$ with age was observed in the inferior fronto-occipital fasciculus (range: 4-30, Asato et al. 2010; Eluvathingal et al. 2007; Krogsrud et al. 2016), in the superior fronto-occipital fasciculus (range: 4-30, Krogsrud et al. 2016; Simmonds et al. 2014), in the superior longitudinal fasciculus (range: 2 months-30 years, Asato et al. 2010; Bava et al. 2010; Krogsrud et al. 2016; Simmonds et al. 2014; Uda et al. 2015) and in the uncinate fasciculus (range: 4-30, Asato et al. 2010; Eluvathingal et al. 2007; Krogsrud et al. 2016; Simmonds et al. 2014). Contrarily, no changes in $\mathrm{RD}$ values were observed between late-adolescent individuals and early-adults (range: 14-32, Bava et al. 2010; Lebel and Beaulieu 2011) in the inferior fronto-occipital fasciculus, superior fronto-occipital fasciculus, superior longitudinal fasciculus, and uncinate fasciculus.

Regarding $\mathrm{AD}$, reduced values across age were found in the inferior fronto-occipital fasciculus (range: 5-17, Eluvathingal et al. 2007; Lebel and Beaulieu 2011), in the superior fronto-occipital fasciculus (range: 8-29, Simmonds et al. 2014), in the superior longitudinal fasciculus (range: 2 months-30 years, Lebel and Beaulieu 2011; Simmonds et al. 2014; Uda et al. 2015), and in the uncinate fasciculus (range: 4-14, Krogsrud et al. 2016; Lebel and Beaulieu 2011); differently, increased AD (range: 8-32) was found in the uncinate fasciculus (Lebel and Beaulieu 2011; Simmonds et al. 2014) and in the inferior fronto-occipital fasciculus and superior longitudinal fasciculus (range: 14-32, Lebel and Beaulieu 2011). However, no changes in AD were observed between childhood (range: 4-9) and early adolescence (range: 5-11) in the inferior fronto-occipital fasciculus (Krogsrud et al. 2016).

Additionally, inter-hemispheric variances were observed in the inferior fronto-occipital fasciculus. Increased MD and $\mathrm{AD}$ were found in the right inferior fronto-occipital fasciculus while both measures were reduced in the left inferior fronto-occipital fasciculus (Bava et al. 2010).

Distinct maturation timings have also been observed in some of the prefrontal tracts. Specifically, non-linear maturation trajectories (increased FA, and decreased MD and RD) were found in the inferior fronto-occipital fasciculus and in the superior fronto-occipital fasciculus. The maturation trajectory of the inferior fronto-occipital fasciculus was illustrated by an exponential growth curve that peaked at the end of the adolescence, and reached a plateau during early adulthood (Bava et al. 2010; Krogsrud et al. 2016; Lebel and Beaulieu 2011; Lebel et al. 2008). The maturation of the superior fronto-occipital fasciculus, as revealed by increased FA and decreased RD and AD, was attained in early adolescence (Krogsrud et al. 2016; Lebel and Beaulieu 2011; Simmonds et al. 2014). An exponential curve of maturation was also observed in the 
superior longitudinal fasciculus, as revealed by increased FA, and decreased MD, RD and AD. The maturational peak was found during adolescence and the plateau was reached throughout early adulthood (Bava et al. 2010; Krogsrud et al. 2016; Lebel and Beaulieu 2011; Lebel et al. 2008; Simmonds et al. 2014); however, another study had found a linear growth of the right superior longitudinal fasciculus FA in young adults (range: 23 to 42, Giorgio et al. 2008). Considering the developmental course of the uncinate fasciculus, it was observed to continue beyond the age 28 , as measured through increased FA and $\mathrm{AD}$, and decreased $\mathrm{MD}$; however in relation to $\mathrm{RD}$, it was fully matured at early adolescence (range: 11-13), (Krogsrud et al. 2016; Lebel and Beaulieu 2011; Simmonds et al. 2014). Yet, one study had revealed a linear developmental trajectory of FA until age 25 (Lebel et al. 2008). In accordance, Muftuler et al. (2012) have found that the lowest rate of change of FA and MD, approximately 0.5 to $1 \%$ per year, measured in the inferior fronto-occipital fasciculus, superior longitudinal fasciculus and uncinate fasciculus, is observed in youth (age range: 6-11). Furthermore, the greatest increase in FA, in addition to MD, AD and RD decrease was observed between birth and 6 years old, in the superior longitudinal fasciculus. The percentage of growth after 6 years old was near zero and $90 \%$ of the maximum FA and MD values were reached around 16 and 9 years old, respectively (Uda et al. 2015).

Finally, regarding gender differences, we observed some variations in the maturation timings of the frontal tracts, between females and males. Specifically, lower $\mathrm{RD}$ values were observed in the females' inferior frontooccipital fasciculus, superior longitudinal fasciculus and uncinate fasciculus (range: 6-17, Asato et al. 2010; Eluvathingal et al. 2007) when compared to males. An exception was observed in the superior longitudinal fasciculus. The frontal portion showed ongoing maturation until early adulthood in the female group; while in males just the parietal portion was fully matured by adolescence (range: 13-17). In the males' group, all the other tracts remained maturing throughout young adulthood (Asato et al. 2010). Additionally, higher FA values were found in the males' superior longitudinal fasciculus and uncinate fasciculus, compared to females; and increased MD was found in the females' superior fronto-occipital fasciculus in comparison to its males' counterparts (range: 5-32, Lebel and Beaulieu 2011).

Despite, other studies found no gender differences in the developmental trajectory of frontal white matter and its association tracts (Bartzokis et al. 2012; Bava et al. 2010; Giorgio et al. 2008; Krogsrud et al. 2016; Kumar et al. 2012; Muftuler et al. 2012; Tamnes et al. 2010; Uda et al. 2015).

\section{Discussion}

The purpose of this review was to systematize the current knowledge on the developmental pathway of the frontal cortex in terms of its microstructural connectivity, as shown by DTI metrics. Overall, the findings suggest an extended developmental course of frontal white matter and its main association tracts (superior longitudinal fasciculus, inferior fronto-occipital fasciculus, superior fronto-occipital fasciculus and uncinate fasciculus), as shown by variations in white mater-related indexes (FA, MD, RD and AD). Furthermore, the majority of the studies suggest linear age-related changes observed in FA MD, RD and AD, since birth until adulthood. Despite, some periods of steadiness may also occur during development.

The results of this systematic review documented that frontal white matter maturation continues far beyond the $3 \mathrm{rd}$ decade of life, which is consistent with other MRI derived measures, as the reduction of cortical thickness and increasing white matter volume observed throughout adolescence and early adulthood (Tamnes et al. 2010). Specifically, a tendency to increase anisotropy (FA) and decrease diffusivity (MD and RD) as a function of age was observed in the frontal white matter and association tracts. In accordance, the lowest FA values and the highest MD and RD values were observed in infants and children; contrastingly, the highest FA and lowest MD and RD were found in young adults. Furthermore, in comparison to all other brain areas, the lowest FA and highest MD were found in the neonatal frontal lobes (Schneider et al. 2004); whereas the highest FA and lowest MD were observed in the adolescents (>144 months) and adult frontal regions, possibly reflecting the protracted myelination of the frontal lobes (Schneider et al. 2004). The process of myelination, axonal density and organization and coherence of fibers' orientation within the frontal white matter seem to last until the thirties, as shown by these studies, documenting a linear increase of FA and decrease in MD and $\mathrm{RD}$, but not so consistently AD until this age range. As mentioned earlier, higher values of anisotropy (e.g., FA) are commonly associated with axonal organization, bundles density, coherence of fibers' orientation (Beaulieu 2002; Ding et al. 2013; Scholz et al. 2009), while lower values of diffusivity seem to be related with intact healthy axons (MD and AD; Beaulieu 2002; Preziosa et al. 2017; Sun et al. 2008) and myelin growth (as measured through RD; Alexander et al. 2007; Song et al. 2003; Sun et al. 2006).

Overall, these studies converge in showing that these DTI metrics are sensitive to different aspects of white matter maturation. It is important to note that although greater axonal organization and coherence are events commonly associated to anisotropic diffusion, it is difficult to attribute particular microstructural features to changes in the fontal white matter FA, as it is not possible to know whether FA variation 
occurs due to the improved coherence of the fiber tracts, or if it solely results from the myelination process. Still, healthy cellular membranes are supposed to be the major basis of the anisotropic water diffusion in white matter fibers, and myelination is thought to modulate the degree of anisotropic diffusion, even though the currently available methods do not allow dissociating these two microstructural features (Beaulieu 2002). For RD, the available literature suggests that decreased values reflect higher myelination, as myelin sheaths seem to modulate anisotropic diffusion by creating barriers to the displacement of water molecules (Beaulieu 2002) with disruptions of the myelin sheaths being related to increasing RD (Song et al. 2002). Finally, the understanding of the $\mathrm{AD}$ measure is more controversial in the literature. While lower $\mathrm{AD}$ values are commonly associated with injured axons (Beaulieu 2002; Sun et al. 2008), they may also be related with regulation of axon branching across development (Gallo 2011; Gibson and Ma 2011), increasing number of white matter tracts or increasing axonal caliber across development and consequent inter-axonal space decrease (Beaulieu 2002; Hagmann et al. 2006; Pierpaoli et al. 1996).

Globally, the studies also suggest that the rate of change in anisotropic and diffusion measures appears to be lower in the interval period between 6 and 11 years old (Muftuler et al. 2012; Qiu et al. 2008; Uda et al. 2015). This deceleration with age (between 6 and 11 years) suggests that this developmental period might rather represent a steady period in frontal white matter development, compared to the first years of life (until 4 years old), (Krogsrud et al. 2016) and adolescence. A possible explanation for white matter growth to slowdown in this age interval may be associated with the observed GM increase (Giedd et al. 1999). In fact, a relation between these two events might eventually exist since GM growth seems to peak around age 12 (followed by a decline), yet the cerebral volume appears to remain quite unchangeable after age 6, as shown by MRI studies (Giedd et al. 1999; Giedd 2004; Gogtay et al. 2004; Lenroot and Giedd 2006). In addition, frontal cortical thickness was found to be negatively correlated with the white matter volume and FA values, and positively associated with $\mathrm{MD}, \mathrm{AD}$ and $\mathrm{RD}$ values (Tamnes et al. 2010). Moreover, distinct timings of maturation were observed in the main frontal association tracts. Specifically, some of the tracts were fully matured between adolescence and early adulthood, either in terms of axonal organization (increased FA and decreased MD and $\mathrm{AD}$ ) or myelin growth (decreased RD), as the inferior fronto-occipital fasciculus (Asato et al. 2010; Bava et al. 2010; Eluvathingal et al. 2007; Lebel and Beaulieu 2011), the superior fronto-occipital fasciculus (Simmonds et al. 2014), and the superior longitudinal fasciculus (Asato et al. 2010; Bava et al. 2010; Lebel et al. 2008); whereas the uncinate fasciculus continued to mature until the thirties (Lebel et al. 2008; Simmonds et al. 2014). These studies suggested that the myelination of the frontal association tracts (i.e., the fronto-occipital, fronto-parietal and fronto-temporal connections) is practically completed by the end of adolescence (Asato et al. 2010; Lebel and Beaulieu 2011; Simmonds et al. 2014); despite the axons' organization continues throughout adulthood (Lebel and Beaulieu 2011; Lebel et al. 2008). This suggests that myelination is likely to be an ongoing process, which occurs since neonatal ages until late adolescence/early adulthood, while fibers' organization and refinement of axonal connectivity might occur at later ages, since this might be a more complex and demanding process, highly dependent on the individual' interplay with the environment.

The current systematic review offers a characterization of the developmental pathway of frontal white matter, as shown by DTI measures, since neonatal ages until early adulthood. Significant alterations of the DTI indexes measured in the frontal white matter of healthy individuals were observed along the brain development, in parallel with alterations in the fibers' pathways and underlying connectivity. This microstructural remodeling suggests that the frontal regions are in constant reorganization and refinement in response to continuous adaptation to environmental stimulation. Moreover, since the diffusion parameters are sensitive to age-dependent fluctuations, the observed DTI metrics changes across the lifecycle suggest that specific, and possibly non-linear, periods of white matter development exist and that changes in frontal white matter microstructure may be qualitatively distinctive in different developmental periods. As highlighted before, the frontal white matter maturation follows a hierarchical trajectory as changes appear to be more pronounced in infancy, between birth and 6 years old, which is followed by a plateau during childhood-early adolescence (range: 6-10), returning to rise between adolescence and early adulthood, that occur in parallel with improvements in cognitive abilities (Gogtay et al. 2004), and in response to the emergent environmental exigencies at each developmental momentum. For example, brain development between adolescence and early adulthood is associated with increasingly new, complex and highly demanding experiences, such as advanced education, employment, new social relations, new familiar responsibilities, that will ultimately influence and be influenced by brain structural and functional architecture, such is the case of higher level executive functions. Furthermore, associations between DTI metrics of the frontal regions and performance in executive functions-related tasks were previously reported, showing an association between performance and anisotropy values and/or diffusivity measures (Madsen et al. 2010; Nagy et al. 2004; Urger et al. 2015). Specifically, higher FA and lower $\mathrm{RD}$ in inferior frontal gyrus of young adolescents were associated to better scores in response inhibition, as measured by 
the Stop Signal Task (Madsen et al. 2010). Working memory capacity was also positively correlated with FA in the superior and inferior frontal gyri in a group of adolescents (Nagy et al. 2004). Finally, anisotropic values were positively associated with executive set-shifting and attentional tasks, and diffusivity values were negatively correlated with inhibitory control, suggesting a link between maturation processes and enhanced cognitive functions (Urger et al. 2015). These findings suggest that the microstructural maturation of the frontal white matter tracts, in particular during adolescence, is likely to underlie executive functions performance. In addition to great developmental improvements, adolescence is also a vulnerable period for external factors that negatively affect mainly the frontal cortex. Therefore, it is often a period for the onset of psychopathological disorders and emergence of maladaptive behaviors (for a review see López-Caneda et al. 2014 in binge drinking) that might be predicted by disrupted developmental processes or interfere with the optimal process of brain maturation.

To conclude, although water diffusion related measures might reflect brain's microstructural connectivity, some limitations should be considered. In particular, DTI measures are not intrinsic properties of the tissues but instead, assumptions driven by a conglomerate of knowledge derived from several fields (mathematics, physics, engineering, computer science and neurosciences) that might limit the associations between DTI data and biological variables. Building on this, Jones and Cercignani (2010) discussed several limitations of the methodology. These limitations can be encountered in each step of the DTI pipeline; i.e., image acquisition, preprocessing, tensor estimation or extraction of scalar measures. For example, distortions caused by eddy currents and head motion, defining the most suitable model to diffusion tensor estimation, or the best method to extract the quantitative measures; e.g., ROI, histogram, voxel-based analysis or TBSS. Anyhow, what seems to be the most noticeable handicap of all is the reliability degree that one may have, when interpreting the DTI metrics (FA, MD, RD and AD) in the light of cerebral development and its association with biological processes (Jones and Cercignani 2010; Soares et al. 2013). Taking into account all of these factors and the basic assumption that DTI is grounded in the manipulation of proton spins and not a direct measure of axonal connectivity, it seems to be worth reflecting on the DTI metrics and accept the fact that errors might be present in the data, as much as in other imaging methods. However, until know, DTI appears to be the most reliable imaging method to assess white matter structure in vivo (Neil et al. 2002).

Finally, while restricting our review to DTI, reports that used other diffusion imaging methods (e.g., DSI or HARDI) were excluded, which could add further important information about frontal white matter maturation. Future reviews should focus in the remaining diffusion methods.

\section{Conclusion}

In this systematic review we highlight the extended course of frontal white matter maturation, evidenced by DTI measures, and documented that brain development is a continuous long-lasting process, driven by specific developmental events (e.g. synaptic refinement, pruning, myelination), designed to sustain the optimal functioning of neuronal networks that support cognition and behavior. Detailed knowledge about the frontal white matter maturation timing is not only important to understand the development of cognitive functions that are dependent on frontal brain regions but it may also be useful in signaling individuals at risk for developmental disorders.

\section{Compliance with ethical standards}

Funding This work was conducted at Psychology Research Centre (UID/PSI/01662/2013), University of Minho, and supported by the Portuguese Foundation for Science and Technology and the Portuguese Ministry of Education and Science through national funds and co-financed by FEDER through COMPETE2020 under the PT2020 Partnership Agreement (POCI-01-0145-FEDER-007653). Sónia Silva Sousa was supported by the SFRH/BD/ 88628/2012, Doctoral Fellowship of the Portuguese Foundation for Science and Technology, co-financed by POPH/FSE through QREN.

Conflict of interest The authors declare no competing financial interests.

Ethical approval This article does not contain any studies with human participants or animals performed by any of the authors.

\section{References}

Alexander, A. L., Lee, J. E., Lazar, M., \& Field, A. S. (2007). Diffusion tensor imaging of the brain. Neurotherapeutics, 4(3), 316-329.

Alger, J. R. (2012). The diffusion tensor imaging toolbox. The Journal of Neuroscience, 32(22), 7418-7428. https://doi.org/10.1523/ JNEUROSCI.4687-11.2012.

Asato, M. R., Terwilliger, R., Woo, J., \& Luna, B. (2010). White matter development in adolescence: a DTI study. Cerebral Cortex, 20, 2122-2131. https://doi.org/10.1093/cercor/bhp282.

Ashtari, M., Cervellione, K. L., Hasan, K. M., Wu, J., McIlree, C., Kester, H., Ardekani, B. A., Roofeh, D., Szeszko, P. R., \& Kumra, S. (2007). White matter development during late adolescence in healthy males: a cross- sectional diffusion tensor imaging study. NeuroImage, 35(2), 501-510.

Assaf, Y., \& Pasternak, O. (2008). Diffusion tensor imaging (DTI)based white matter mapping in brain research: a review. Journal of Molecular Neuroscience, 34(1), 51-61. https://doi.org/10.1007/ s12031-007-0029-0.

Aung, W. Y., Mar, S., \& Benzinger, T. L. (2013). Diffusion tensor MRI as a biomarker in axonal and myelin damage. Imaging in Medicine, 5(5), 427-440. https://doi.org/10.2217/iim.13.49.

Barnea-Goraly, N., Menon, V., Eckert, M., Tamm, L., Bammer, R., Karchemskiy, A., Dant, C. C., \& Reiss, A. L. (2005). White matter development during childhood and adolescence: a 
cross- sectional diffusion tensor imaging study. Cerebral Cortex, 15, 1848-1854.

Bartzokis, G., Lu, P. H., Heydari, P., Couvrette, A., Lee, G. J., Kalashyan, G., Kalashyan, G., Freeman, F., Grinstead, J. W., Villablanca, P., Finn, J. P., Mintz, J., Alger, J. R., \& Altshuler, L. L. (2012). Multimodal magnetic resonance imaging assessment of white matter aging trajectories over the lifespan of healthy individuals. Biological Psychiatry, 72, 1026-1034.

Bava, S., Thayer, R., Jacobus, J., Ward, M., Jernigan, T. L., \& Tapert, S. F. (2010). Longitudinal characterization of white matter maturation during adolescence. Brain Research, 1327, 38-46.

Beaulieu, C. (2002). The basis of anisotropic water diffusion in the nervous system - a technical review. NMR in Biomedicine, 15(7-8), 435-455. https://doi.org/10.1002/nbm.782.

Bennett, E. L., Diamond, M. C., Krech, D., \& Rosenzweig, M. R. (1964). Chemical and anatomical plasticity of brain: changes in brain through experience, demanded by learning theories, are found in experiments with rats. Science, 146(3644), 610-619. https://doi.org/10.1126/science.146.3644.610.

Bonekamp, D., Nagae, L. M., Degaonkar, M., Matson, M., Abdall, W. M. A., Barker, P. B., Mori, S., \& Horská, A. (2007). Diffusion tensor imaging in children and adolescents: reproducibility, hemispheric, and age-related differences. NeuroImage, 34(2), 733-742.

Casey, B. J., Giedd, J. N., \& Thomas, K. M. (2000). Structural and functional brain development and its relation to cognitive development. Biological Psychology, 54(1-3), 241-257. https://doi. org/10.1016/S0301-0511(00)00058-2.

Chilla, G. S., Tan, C. H., Xu, C., \& Poh, C. L. (2015). Diffusion weighted magnetic resonance imaging and its recent trend-a survey. Quantitative Imaging in Medicine and Surgery, 5(3), 407422. https://doi.org/10.3978/j.issn.2223-4292.2015.03.01.

Colby, J. B., Van Horn, J. D., \& Sowell, E. R. (2011). Quantitative in vivo evidence for broad regional gradients in the timing of white matter maturation during adolescence. NeuroImage, 54(1), 25-31. https://doi.org/10.1016/j.neuroimage.2010.08.014.

Deoni, S. C. L., Mercure, E., Blasi, A., Gasston, D., Thomson, A., Johnson, M., Williams, S. C., \& Murphy, D. G. M. (2011). Mapping infant brain myelination with magnetic resonance imaging. The Journal of Neuroscience, 31(2), 784-791.

Ding, A. Y., Li, Q., Zhou, I. Y., Ma, S. J., Tong, G., McAlonan, G. M., \& Wu, E. X. (2013). MR diffusion tensor imaging detects rapid microstructural changes in amygdala and hippocampus following fear conditioning in mice. PLoS One, 8(1), e51704. https://doi. org/10.1371/journal.pone.0051704.

Dubois, J., Dehaene-Lambertz, G., Kulikova, S., Poupon, C., Huppi, P. S., \& Hertz-Pannier, L. (2013). The early development of brain white matter: a review of imaging studies in fetuses, newborns and infants. Neuroscience, 276, 48-71. https://doi.org/10.1016/j. neuroscience.2013.12.044

Durston, S., Hulshoff Pol, H. E., Casey, B. J., Giedd, J. N., Buitelaar, J. K., \& van Engeland, H. (2001). Anatomical MRI of the developing human brain: what have we learned? Journal of the American Academy of Child and Adolescent Psychiatry, 40(9), 1012-1020.

Eluvathingal, T. J., Hasan, K. M., Kramer, L., Fletcher, J. M., \& EwingCobbs, L. (2007). Quantitative diffusion tensor tractography of association and projection fibers in normally developing children and adolescents. Cerebral Cortex, 17, 2760-2768.

Fields, R. D. (2005). Myelination: an overlooked mechanism of synaptic plasticity? The Neuroscientist, 11(6), 528-531. https://doi. org/10.1177/1073858405282304.

Fields, R. D. (2008). White matter in learning, cognition and psychiatric disorders. Trends in Neurosciences, 31(7), 361-370.

Fox, R. J., Cronin, T., Lin, J., Wang, X., Sakaie, K., Ontaneda, D., Mahmoud, S. Y., Lowe, M. J., \& Phillips, M. D. (2011). Measuring myelin repair and axonal loss with diffusion tensor imaging.
AJNR. American Journal of Neuroradiology, 32(1), 85-91. https:// doi.org/10.3174/ajnr.A2238.

Froeling, M., Pullens, P., \& Leemans, A. (2016). DTI analysis methods: region of interest analysis. pp. 175-182. https://doi. org/10.1007/978-1-4939-3118-7_9.

Gallo, G. (2011). The cytoskeletal and signaling mechanisms of axon collateral branching. Developmental Neurobiology, 71(3), 201220. https://doi.org/10.1002/dneu.20852.

Gibson, D. A., \& Ma, L. (2011). Developmental regulation of axon branching in the vertebrate nervous system. Development, 138(2), 183-195. https://doi.org/10.1242/dev.046441.

Giedd, J. N. (2004). Structural magnetic resonance imaging of the adolescent brain. Annals of the New York Academy of Sciences, 1021, 77-85. https://doi.org/10.1196/annals.1308.009.

Giedd, J. N. (2008). The teen brain: insights from neuroimaging. The Journal of Adolescent Health, 42(4), 335-343. https://doi. org/10.1016/j.jadohealth.2008.01.007.

Giedd, J. N., \& Rapoport, J. L. (2010). Structural MRI of pediatric brain development: what have we learned and where are we going? Neuron, 67(5), 728-734. https://doi.org/10.1016/j. neuron.2010.08.040.

Giedd, J. N., Blumenthal, J., Jeffries, N. O., Castellanos, F. X., Liu, H., Zijdenbos, A., Paus, T., Evans, A. C., \& Rapoport, J. L. (1999). Brain development during childhood and adolescence: a longitudinal MRI study. Nature Neuroscience, 2, 861-863.

Giedd, J. N., Clasen, L. S., Lenroot, R., Greenstein, D., Wallace, G. L., Ordaz, S., Molloy, E. A., Blumenthal, J. D., Tossell, J. W., Stayer, C., Samango-Sprouse, C. A., Shen, D., Davatzikos, C., \& Chrousos, G. P. (2006). Puberty-related influences on brain development. Molecular and Cellular Endocrinology, 254-255, 154-162. https://doi.org/10.1016/j.mce.2006.04.016.

Giorgio, A., Watkins, K. E., Douaud, G., James, A. C., James, S., De Stefano, N., Matthews, P. M., Smith, S. M., \& Johansen-Berg, H. (2008). Changes in white matter microstructure during adolescence. NeuroImage, 39, 52-61.

Gogtay, N., Giedd, J. N., Lusk, L., Hayashi, K. M., Greenstein, D., Vaituzis, A. C., Nugent, T. F. 3rd, Herman, D. H., Clasen, L. S., Toga, A. W., Rapoport, J. L., \& Thompson, P. M. (2004). Dynamic mapping of human cortical development during childhood through early adulthood. Proceedings of the National Academy of Sciences of the United States of America, 101(21), 8174-8179.

Hagmann, P., Jonasson, L., Maeder, P., Thiran, J.-P., Wedeen, V. J., \& Meuli, R. (2006). Understanding diffusion MR imaging techniques: from scalar diffusion-weighted imaging to diffusion tensor imaging and beyond. RadioGraphics, 26, 205-223.

Hasan, K. M., Alexander, A. L., \& Narayana, P. A. (2004). Does fractional anisotropy have better noise immunity characteristics than relative anisotropy in diffusion tensor MRI? An analytical approach. Magnetic Resonance in Medicine, 51, 413-417.

Hasan, K. M., Sankar, A., Halphen, C., Kramer, L. A., Brandt, M. E., Juranek, J., Cirino, P. T., Fletcher, J. M., Papanicolaou, A. C., \& Ewing-Cobbs, L. (2007). Development and organization of the human brain tissue compartments across the lifespan using diffusion tensor imaging. Neuroreport, 18(16), 1735-1739.

Huttenlocher, P. R. (1979). Synaptic density in human frontal cortexdevelopmental changes and effects of aging. Brain Research, 163, 195-205.

Jones, D. K., \& Cercignani, M. (2010). Twenty-five pitfalls in the analysis of diffusion MRI Data. NMR in Biomedicine, 23, 803-820.

Klingberg, T., Vaidya, C. J., Gabrieli, J. D., Moseley, M. E., \& Hedehus, M. (1999). Myelination and organization of the frontal white matter in children: a diffusion tensor MRI study (Abstract). Neuroreport, 10(13), 2817-2821.

Kraus, M. F., Susmaras, T., Caughlin, B. P., Walker, C. J., Sweeney, J. A., \& Little, D. M. (2007). White matter integrity and cognition 
in chronic traumatic brain injury: a diffusion tensor imaging study. Brain, 130, 2508-2519.

Krogsrud, S. K., Fjell, A. M., Tamnes, C. K., Grydeland, H., Mork, L., Due-Tonnessen, P., Biornerud, A., Sampaio-Baptista, C., Anderson, J., Johansen-Berg, H., \& Walhovd, K. B. (2016). Changes in white matter microstructure in the developing brain-a longitudinal diffusion tensor imaging study of children from 4 to 11 years of age. NeuroImage, 124(Pt A), 473-486. https://doi. org/10.1016/j.neuroimage.2015.09.017.

Kumar, R., Nguyen, H. D., Macey, P. M., Woo, M. A., \& Harper, R. M. (2012). Regional brain axial and radial diffusivity changes during development. Journal of Neuroscience Research, 90(2), 346-355.

Le Bihan, D. (2003). Looking into the functional architecture of the brain with diffusion MRI. Nature, 4, 469-480.

Le Bihan, D., Mangin, J.-F., Poupon, C., Clark, C. A., Pappata, S., Molko, N., \& Chabriat, H. (2001). Diffusion tensor imaging: concepts and applications. Journal of Magnetic Resonance Imaging, $13,534-546$

Lebel, C., \& Beaulieu, C. (2011). Longitudinal development of human brain wiring continues from childhood into adulthood. The Journal of Neuroscience, 31(30), 10937-10947.

Lebel, C., Walker, L., Leemans, A., Phillips, L., \& Beaulieu, C. (2008). Microstructural maturation of the human brain from childhood to adulthood. NeuroImage, 40, 1044-1055.

Lenroot, R. K., \& Giedd, J. N. (2006). Brain development in children and adolescents: Insights from anatomical magnetic resonance imaging. Neuroscience and Biobehavioral Reviews, 30, 718-729.

Li, T. Q., \& Noseworthy, M. D. (2002). Mapping the development of white matter tracts with diffusion tensor imaging. Developmental Science, 5(3), 293-300.

Lobel, U., Sedlacik, J., Gullmar, D., Kaiser, W. A., Reichenbach, J. R., \& Mentzel, H. J. (2009). Diffusion tensor imaging: the normal evolution of ADC, RA, FA, and eigenvalues studied in multiple anatomical regions of the brain. Neuroradiology, 51(4), 253-263. https://doi.org/10.1007/s00234-008-0488-1.

Lopez-Caneda, E., Mota, N., Crego, A., Velasquez, T., Corral, M., Holguín, S. R., \& Cadaveira, F. (2014). Neurocognitive anomalies associated with the binge drinking pattern of alcohol consumption in adolescents and young people: a review. Adicciones, 26(4), 334-359.

Madsen, K. S., Baaré, W.F.C., Vestergaard, M., Skimminge, A., Ejersbo, L. R., Ramsøy, T. Z., Gerlach, C., Akeson, P., Paulson, O. B., \& Jernigan, T. L. (2010). Response inhibition is associated with white matter microstructure in children. Neuropsychologia, $48,854-862$

Martino, J., De Witt Hamer, P. C., Berger, M. S., Lawton, M. T., Arnold, C. M., de Lucas, E. M., \& Duffau, H. (2013). Analysis of the subcomponents and cortical terminations of the perisylvian superior longitudinal fasciculus: a fiber dissection and DTI tractography study. Brain Structure and Function, 218, 105-121.

Medana, I. M., \& Esiri, M. M. (2003). Axonal damage: a key predictor of outcome in human CNS diseases. Brain, 126, 515-530.

Moher, D., Liberati, A., Tetzlaff, J., Altman, D. G., \& The PRISMA Group (2009). Preferred reporting items for systematic reviews and meta-analyses: the PRISMA statement. Annals of Internal Medicine, 151(4), 264-269.

Moon, W.-J., Provenzale, J., Sarikaya, B., Ihn, Y. K., Morlese, J., Chen, S., \& DeBellis, M. D. (2011). Diffusion tensor imaging assessment of white matter maturation in childhood and adolescence. American Journal of Roentgenology, 197(3), 704-712.

Mori, S., \& Zhang, J. (2006). Principles of diffusion tensor imaging and its applications to basic neuroscience research. Neuron, 51(5), 527-539. https://doi.org/10.1016/j.neuron.2006.08.012.

Muftuler, L. T., Davis, E. P., Buss, C., Solodkin, A., Su, M. Y., Head, K. M., Hasso, A. N., \& Sandman, C. A. (2012). Development of white matter pathways in typically developing preadolescent children. Brain Research, 1466, 33-43. https:// doi.org/10.1016/j.brainres.2012.05.035.

Mukherjee, P., Berman, J. I., Chung, S. W., Hess, C. P., \& Henry, R. G. (2008). Diffusion tensor MR imaging and fiber tractography: theoretic underpinnings. AJNR. American Journal of Neuroradiology, 29(4), 632-641. https://doi.org/10.3174/ajnr.A1051.

Nagy, Z., Westerberg, H., \& Klingberg, T. (2004). Maturation of white matter is associated with the development of cognitive functions during childhood. Journal of Cognitive Neuroscience, 16(7), 1227-1233.

Neil, J., Miller, J., Mukherjee, P., \& Huppi, P. S. (2002). Diffusion tensor imaging of normal and injured developing human braina technical review. NMR in Biomedicine, 15(7-8), 543-552. https://doi.org/10.1002/nbm.784.

Nishikawa, R. M., Lützkendorf, R., Whiting, B. R., Hertel, F., Heidemann, R., Thiel, A., Luchtmann, M., Plaumann, M., Stadler, J., Baecke, S., \& Bernarding, J. (2013). Non-invasive high-resolutiontracking of human neuronal pathways: diffusion tensor imaging at 7T with $1.2 \mathrm{~mm}$ isotropic voxel size. 8668,866846 . https://doi.org/10.1117/12.2006764.

Paus, T. (2010). Growth of white matter in the adolescent brain: myelin or axon? Brain and Cognition, 72, 26-35.

Paus, T., Zijdenbos, A., Worsley, K., Collins, D. L., Blumenthal, J., Giedd, J. N., Rapoport, J. L., \& Evans, A. C. (1999). Structural maturation of neural pathways in children and adolescents: in vivo study. Science, 283(5409), 1908-1911.

Paus, T., Collins, D. L., Evans, A. C., Leonard, G., Pike, B., \& Zijdenbos, A. (2001). Maturation of white matter in the human brain: a review of magnetic resonance studies. Brain Research Bulletin, 54(3), 255-266.

Petanjek, Z., Judaš, M., Šimić, G., Rašin, M. R., Uylings, H. B. M., Rakic, P., \& Kostovića, I. (2011). Extraordinary neoteny of synaptic spines in the human prefrontal cortex. Proceedings of the National Academy of Sciences of the United States of America, 108(32), 13281-13286. https://doi.org/10.1073/ pnas. 1105108108.

Pierpaoli, C., \& Basser, P. J. (1996). Toward a quantitative assessment of diffusion anisotropy. Magnetic Resonance in Medicine, 36(6), 893-906. https://doi.org/10.1002/mrm.1910360612.

Pierpaoli, C., Jezzard, P., Basser, P. J., Barnett, A., \& Di Chiro, G. (1996). Diffusion tensor MR imaging of the human brain. Radiology, 201, 637-648.

Preziosa, P., Pagani, E., Morelli, M. E., Copetti, M., Martinelli, V., Pirro, F., Falini, A., Comi, G., Filippi, M., \& Rocca, M. A. (2017). DT MRI microstructural cortical lesion damage does not explain cognitive impairment in MS. Multiple Sclerosis. 1352458516689147. https://doi.org/10.1177/1352458516689147.

Qiu, D., Tan, L.-H., Zhou, K., \& Khong, P.-L. (2008). Diffusion tensor imaging of normal white matter maturation from late childhood to young adulthood: voxel-wise evaluation of mean diffusivity, fractional anisotropy, radial and axial diffusivities, and correlation with reading development. NeuroImage, 41, 223-232.

Qiu, M., Li, Q., Liu, G., Xie, B., \& Wang, J. (2010). Voxel-based analysis of white matter during adolescence and young adulthood. Brain \& Development, 32, 531-537.

Roosendaal, S. D., Geurts, J. J., Vrenken, H., Hulst, H. E., Cover, K. S., Castelijns, J. A., Pouwels, J. A., \& Barkhof, F. (2009). Regional DTI differences in multiple sclerosis patients. NeuroImage, 44(4), 1397-1403. https://doi.org/10.1016/j.neuroimage.2008.10.026.

Schmithorst, V. J., Wilke, M., Dardzinski, B. J., \& Holland, S. K. (2005). Cognitive functions correlate with white matter architecture in a normal pediatric population: a diffusion tensor MRI study. Human Brain Mapping, 26(2), 139-147. https://doi. org/10.1002/hbm.20149.

Schneider, J.F.L., Il'yasov, K. A., Hennig, J., \& Martin, E. (2004). Fast quantitative diffusion-tensor imaging of cerebral white matter 
from the neonatal period to adolescence. Neuroradiology, 46, 258-266.

Scholz, J., Klein, M. C., Behrens, T. E., \& Johansen-Berg, H. (2009). Training induces changes in white-matter architecture. Nature Neuroscience, 12(11), 1370-1371. https://doi.org/10.1038/ nn.2412.

Shaw, P., Kabani, N. J., Lerch, J. P., Eckstrand, K., Lenroot, R., Gogtay, N., Greenstein, D., Clasen, L., Evans, A., Rapoport, J. L., Giedd, J. N., \& Wise, S. P. (2008). Neurodevelopmental trajectories of the human cerebral cortex. The Journal of Neuroscience, 28(14), 3586-3594. https://doi.org/10.1523/JNEUROSCI.5309-07.2008.

Simmonds, D. J., Hallquist, M. N., Asato, M., \& Luna, B. (2014). Developmental stages and sex differences of white matter and behavioral development through adolescence: a longitudinal diffusion tensor imaging (DTI) study. NeuroImage, 92, 356-368. https://doi.org/10.1016/j.neuroimage.2013.12.044.

Smith, S. M., Jenkinson, M., Johansen-Berg, H., Rueckert, D., Nichols, T. E., Mackay, C. E., Watkins, K. E., Ciccarelli, O., Cader, M. Z., Matthews, P. M., \& Behrens, T. E. (2006). Tract-based spatial statistics: voxelwise analysis of multi-subject diffusion data. NeuroImage, 31(4), 1487-1505. https://doi.org/10.1016/j. neuroimage.2006.02.024.

Snaidero, N., \& Simons, M. (2014). Myelination at a glance. Journal of Cell Science, 127, 2999-3004.

Snook, L., Paulson, L.-A., Roy, D., Phillips, L., \& Beaulieu, C. (2005). Diffusion tensor imaging of neurodevelopment in children and young adults. NeuroImage, 26, 1164-1173.

Snook, L., Plewes, C., \& Beaulieu, C. (2007). Voxel based versus region of interest analysis in diffusion tensor imaging of neurodevelopment. NeuroImage, 34, 243-252.

Soares, J. M., Marques, P., Alves, V., \& Sousa, N. (2013). A hitchhiker's guide to diffusion tensor imaging. Frontiers in Neuroscience, 7, 31. https://doi.org/10.3389/fnins.2013.00031.

Song, S. K., Sun, S. W., Ramsbottom, M. J., Chang, C., Russell, J., \& Cross, A. H. (2002). Dysmyelination revealed through MRI as increased radial (but unchanged axial) diffusion of water. NeuroImage, 17(3), 1429-1436. https://doi.org/10.1006/ nimg.2002.1267.

Song, S.-K., Sun, S.-W., Ju, W.-K., Lin, S.-J., Cross, A. H., \& Neufeld, A. H. (2003). Diffusion tensor imaging detects and differentiates axon and myelin degeneration in mouse optic nerve after retinal ischemia. Neurolmage, 20, 1714-1722.
Sun, S. W., Liang, H. F., Trinkaus, K., Cross, A. H., Armstrong, R. C., \& Song, S. K. (2006). Noninvasive detection of cuprizone induced axonal damage and demyelination in the mouse corpus callosum. Magnetic Resonance in Medicine, 55(2), 302-308. https://doi. org/10.1002/mrm.20774.

Sun, S.-W., Liang, H.-F., Cross, A. H., \& Song, S.-K. (2008). Evolving Wallerian degeneration after transient retinal ischemia in mice characterized by diffusion tensor imaging. NeuroImage, 40(1), 1-10. https://doi.org/10.1016/j.neuroimage.2007.11.049.

Taki, Y., Thyreau, B., Hashizume, H., Sassa, Y., Takeuchi, H., Wu, K., Kotozaki, Y., Nouchi, R., Asano, K., Fukuda, H., \& Kawashima, R. (2013). Linear and curvilinear correlations of brain white matter volume, fractional anisotropy, and mean diffusivity with age using voxel-based and region-of-interest analyses in 246 healthy children. Human Brain Mapping, 34(8), 1842-1856. https://doi. org/10.1002/hbm.22027.

Tamnes, C. K., Østby, Y., Fjell, A. M., Westlye, L. T., Due-Tønnessen, P., \& Walhovd, K. B. (2010). Brain maturation in adolescence and young adulthood: regional age-related changes in cortical thickness and white matter volume and microstructure. Cerebral Cortex, 20, 534-548.

Thomason, M. E., \& Thompson, P. M. (2011). Diffusion imaging, white matter, and psychopathology. Annual Review of Clinical Psychology, 7, 63-85.

Uda, S., Matsui, M., Tanaka, C., Uematsu, A., Miura, K., Kawana, I., \& Noguchi, K. (2015). Normal development of human brain white matter from infancy to early adulthood: a diffusion tensor imaging study. Developmental Neuroscience, 37(2), 182-194. https://doi. org/10.1159/000373885.

Urger, S. E., De Bellis, M. D., Hooper, S. R., Woolley, D. P., Chen, S. D., \& Provenzale, J. (2015). The superior longitudinal fasciculus in typically developing children and adolescents: diffusion tensor imaging and neuropsychological correlates. Journal of Child Neurology, 30(1), 9-20. https://doi.org/10.1177/0883073813520503.

Westin, C. F., Maier, S. E., Mamata, H., Nabavi, A., Jolesz, F. A., \& Kikinis, R. (2002). Processing and visualization for diffusion tensor MRI. Medical Image Analysis, 6, 93-108.

Xie, M., Wang, Q., Wu, T. H., Song, S. K., \& Sun, S. W. (2011). Delayed axonal degeneration in slow Wallerian degeneration mutant mice detected using diffusion tensor imaging. Neuroscience, 197, 339-347. https://doi.org/10.1016/j. neuroscience.2011.09.042. 